Article

\title{
Some Fixed Point Results on Rectangular Metric-Like Spaces Endowed with a Graph
}

\author{
Nizar Souayah ${ }^{1, *}$ and Mehdi Mrad ${ }^{2}$ \\ 1 Department of Natural Sciences, Community College Al-Riyadh, King Saud University, \\ Riyadh 4545, Saudi Arabia \\ 2 Department of Industrial Ingeneering, College of Engineering, King Saud University, \\ Riyadh 4545, Saudi Arabia; mmrad@ksu.edu.sa \\ * Correspondence: nsouayah@ksu.edu.sa; Tel.: +966-540373511
}

Received: 27 November 2018; Accepted: 20 December 2018; Published: 26 December 2018

\begin{abstract}
The objective of this paper is to establish the existence and uniqueness of fixed points on rectangular metric-like spaces endowed with a graph. We introduce the notion of some generalized $G$-contractions principle. The usefulness of the considered metric space in real work is highlighted. The obtained results generalize some notes in the literature. Some examples are presented to support the main results.
\end{abstract}

Keywords: fixed point; graph theory; rectangular metric-like spaces; G-contraction

\section{Introduction}

Graph theory has been the focus of many researchers which are used in various areas. In fact, graph theoretical concept modeled and analyzed systems in different fields. Mathematicians are becoming aware of the significance of this theory and they combined it with other branches of mathematics. One of the last and important fields were a fixed point theory. In 2006, Espinola and Kirk introduced some results on combining fixed point theory and graph theory [1]. In 2008, Jachymski [2] provided an interesting approach in this direction. His work is considered as a reference in this domain. Starting from this approach, many researchers have discussed the existence and uniqueness of fixed points in different metric spaces with a graph and several contractions that are used in several types of spaces [3-13].

In addition, the fixed point theory on metric spaces endowed with graph represented a favorable theoretical background for the concept of the network nodes. Depending on its application, this concept modeled a large variety of functions in different fields such data communications, internet network nodes, telecommunications network nodes. In order to understand the relationship between the network nodes and the fixed point theory, we consider the case study theme from a communication network. In this field, a network node is a connection point that can receive, create store or send along distributed network routes. Each node can transmit or redistribute data to other network nodes according to a routing strategy. For example, in internet networks, host computers that are identified by an IP address are considered as a physical network node. In data communications, a network node can be data terminal equipment, routers or servers. Hence, by combining all kinds of physical networks, the node is a vector which allowed us to define a network vector space $E$ [14]. This space can be treated as a set of nodes and links. The function of links is to spread data provided by those nodes. Therefore, there exists a characteristic correlation between any two different nodes that can be given by a certain correlation measure $d$. In this respect, we notice that $(E, d)$ is a metric space. Knowing the limited dimension of the space in a real network, we obtain the completeness; then, $E$ is a Banach space. As stated above, we have transmission of data between nodes. More precisely, the transmitted information from a network node $x$ belonging to $E$ to another network node $y$ can be obtained using an 
operator $T$. This operator could be a routing algorithm or a mining algorithm [14]. On the other hand, in real work and taking into account the increasing of the complexity of nodes correlation, we can conclude that the measure between two nodes obtained from mapping by the operator $T$ becomes relatively small. Thus, the network operator $T$ is the compression mapping on $E$ and the compression coefficient is $\alpha$ such that $0<\alpha<1$. Therefore, $T: E \longrightarrow E$ is a contraction mapping on $E$ and all the assumptions of Banach fixed point theorem are made. Then, $T$ has a fixed point $u$ satisfying $T u=u$ and relative to the network nodes; $u$ is the network path prediction target node.

According to what we mention, it is very interesting to investigate in fixed point theory on metric spaces with graphs and to prepare a theoretical framework for applications to real cases using different metrics and contractions that can model different phenomena.

Throughout this paper, we consider the complete rectangular metric-like spaces and we debate fixed point results for generalized G-contractive type mapping. We will introduce the notion of $G_{r}$-Kannan contraction, $G$-graphic contraction and $(G-\phi)$-contraction. Some examples are presented to illustrate the obtained results.

The notion of metric-like is original and very important in real work. Actually, if we consider two nodes $u$ and $v$ such that $u=v$, the measure between them is not necessarily equal to zero, which is the case in several fields. In addition, the rectangular metric that is characterized by its rectangular inequality plays an important role mainly when the number of nodes increases. In fact, to transmit information from source node to a target node, we observe that the shortest path contains in general more than one node.

\section{Preliminaries}

First, we remind the reader of the concept of metric-like spaces as well as the rectangular metric-like spaces and the convergence and completeness in this space. We presented also some basic concepts of graph theory which will be needed in the sequel.

Definition 1. Ref. [15] Let a nonempty set $X$ and $\sigma_{l}: X \times X \longrightarrow \mathbb{R}^{+}$be a mapping satisfying the following conditions

for all $a, b, c \in X$ :

$\left(L_{1}\right) \quad \sigma_{l}(a, b)=0 \Longrightarrow a=b$,

$\left(L_{2}\right) \quad \sigma_{l}(a, b)=\sigma_{l}(b, a)$

$\left(L_{3}\right) \quad \sigma_{l}(a, c) \leq \sigma_{l}(a, b)+\sigma_{l}(b, c)$.

Then, $\sigma_{l}$ is called a metric-like and the pair $\left(X, \sigma_{l}\right)$ determine a metric-like space.

Note that metric-like spaces are more general than both metric and partial metric spaces since $\sigma_{l}(a, a)$ can be different from zero; in addition, $\sigma_{l}(a, a)$ may be bigger than $\sigma_{l}(a, b)$. Thereby, every partial metric space is a metric-like space.

Example 1. Let $X=[0,1]$ and $\sigma_{l}(a, b)=\left\{\begin{array}{lr}3, & \text { if } a=b, \\ 2, & \text { otherwise. }\end{array}\right.$

It is easy to see that $\left(X, \sigma_{l}\right)$ is a metric-like space but not a partial metric space because $\sigma_{l}(0,0) \not \leq \sigma_{l}(0,1)$.

Definition 2. Ref. [16] Let there be a nonempty set $X$ and $\sigma_{r}: X \times X \longrightarrow \mathbb{R}^{+}$a mapping satisfying the following conditions for all $x, y, z, t \in X$ :

$\left(L_{1}\right) \quad \sigma_{r}(x, y)=0 \Longrightarrow x=y$,

$\left(L_{2}\right) \quad \sigma_{r}(x, y)=\sigma_{r}(y, x)$,

(Ls) $\quad \sigma_{r}(x, y) \leq \sigma_{r}(x, z)+\sigma_{r}(z, t)+\sigma_{r}(t, y)$ for all different $z, t \in X \backslash\{x, y\}$ (Rectangular inequality).

Then, $\sigma_{r}$ is called a rectangular metric-like and the pair $\left(X, \sigma_{r}\right)$ determine a rectangular metric-like space. 
Example 2. Let $X=\{1,2,3,4,5\}$ and define the mapping $\sigma_{r}: X \times X \longrightarrow[0, \infty)$ by

$$
\sigma_{r}(x, y)= \begin{cases}2, & \text { for } x \neq y \\ 4, & \text { if } x=y=1 \\ 0, & \text { otherwise. }\end{cases}
$$

It is easy to see that conditions $L_{1}$ and $L_{2}$ are satisfied. Let's verify the rectangular inequality given by $L_{3}$. Considering $x, y \in X$ and for all distinct $u, v \in X \backslash\{x, y\}$, we have

$$
\sigma_{r}(x, u)+\sigma_{r}(u, v)+\sigma_{r}(v, y)=2+\sigma_{r}(u, v)+2=4+\sigma_{r}(u, v) \geq \sigma_{r}(x, y) \forall x, y \in X .
$$

Therefore, $\left(X, \sigma_{r}\right)$ is a rectangular metric-like space.

Definition 3. Ref. [16] Let $\left(X, \sigma_{r}\right)$ be a rectangular metric-like space. Then,

1. A sequence $\left\{x_{n}\right\}$ in $\left(X, \sigma_{r}\right)$ converges to $x \in X$ if and only if $\lim _{n \rightarrow \infty} \sigma_{r}\left(x_{n}, x\right)=\sigma_{r}(x, x)$.

2. A sequence $\left\{x_{n}\right\}$ in $\left(X, \sigma_{r}\right)$ is said to be $\sigma_{r}$-Cauchy sequence if and only if the limit of $\sigma_{r}\left(x_{n}, x_{m}\right)$ exists and finite as $n \rightarrow \infty$.

3. $\left(X, \sigma_{r}\right)$ is said to be $\sigma_{r}$-complete if any $\sigma_{r}$-Cauchy sequence $\left\{x_{n}\right\}$ in $X$ converges to some point $x$ such that

$$
\lim _{n \rightarrow \infty} \sigma_{r}\left(x_{n}, x\right)=\sigma_{r}(x, x)=\lim _{n, m \rightarrow \infty} \sigma_{r}\left(x_{n}, x_{m}\right)
$$

Remark 1. The convergence defined in the last definition is the convergence obtained in the sense of the topology generated by the open balls

$$
B_{\sigma_{r}}(x, \delta)=\left\{y \in X \text { such that } \mid \sigma_{r}(x, y)-\sigma_{r}(x, x)<\delta\right\}, x \in X .
$$

For more details on the topology of rectangular metric-like spaces, we refer to [16].

Next, we present a few basic concepts of graph theory.

According to Jachymski [2], we consider a rectangular metric-like space $\left(X, \sigma_{r}\right)$ and $\Delta$ is the diagonal of $X \times X$. A graph $G$ is defined by the set $V=V(G)$ of vertices coinciding with $X$ and the set $E=E(G)$ of its edges such that $\Delta \subset E(G)$. Assume that the graph $G$ has no parallel edges. Therefore, $G$ can be identified with the pair $(V, E)$.

In addition, the graph $G$ may be considered as a weighted graph by assigning to each edge the distance given by the $\sigma_{r}$-metric between its vertices. We denote by $G^{-1}$ the graph obtained from $G$ by reversing the direction of edges in $G$. Then,

$$
E\left(G^{-1}\right)=\left\{(x, y) \in X^{2}:(y, x) \in E(G)\right\} \text { and } V\left(G^{-1}\right)=V(G)
$$

Let $\tilde{G}$ denote the undirected graph built by ignoring the direction of all edges that is

$$
E(\tilde{G})=E(G) \cup E\left(G^{-1}\right) .
$$

Definition 4. A subgraph is a graph that consists of a subset of a graph's edges and associated vertices.

Definition 5. Consider two vertices $a$ and $b$ in a graph $G$. A path in $G$ from a to b of length $k(k \in \mathbb{N} \cup\{0\})$ is a sequence $\left(x_{i}\right)_{i=0}^{k}$ of $k+1$ distincts vertices such that $x_{0}=a, x_{k}=b$ and $\left(x_{i}, x_{i+1}\right) \in E(G)$ for $i=1,2, \ldots, k$.

Definition 6. 1. A graph $G$ is called connected if there is a path between any two vertices of $G$ and it is weakly connected if $\tilde{G}$ is connected.

2. A graph $G$ is said to be simple if it has neither multiple edges nor loops.

Consider $x$ a vertex in a graph $G$. The subgraph denoted by $G_{x}$ and constituted by all edges and vertices which are contained in some path in $G$ beginning at $x$ is called the component of $G$ containing 
$x$. In this case, $V\left(G_{x}\right)=[x]_{G}$, where $[x]_{G}$ denotes the equivalence class of relation $R$ ( $x R y$ if there is a path in $G$ from $x$ to $y$ ).

In order to apply the rectangular inequality later to the vertices of the graph, we need to consider a graph of length bigger than 2 which means that, between two vertices, we can find a path through at least two other vertices.

\section{Main Results}

In this section, we consider $\left(X, \sigma_{r}\right)$ to be a rectangular metric-like space, and $G$ is a simple graph of length bigger than 2 without parallel edges such that $V(G)=X$. Define Fixf $=\{u \in X / f u=u\}$.

Now, let us define the Kannan operator on metric space $(X, d)$.

Definition 7. Ref. [17] Let $(X, d)$ be a metric space. $f: X \longrightarrow X$ is called a Kannan operator if there exists $a \in\left[0, \frac{1}{2}\right)$ such that :

$$
d(f x, f y) \leq a[d(x, f x)+d(y, f y)] \forall x, y \in X .
$$

Inspired by the Kannan operator, we introduce the concept of $G_{r}$-contraction in rectangular metric-like spaces with a graph.

Definition 8. Let $\left(X, \sigma_{r}\right)$ be a rectangular metric-like space endowed with a graph $G$. The mapping $f: X \longrightarrow$ $X$ is said to be $G_{r}$-Kannan contraction if

1.

$$
\forall x, y \in X, \text { If }(x, y) \in E(G), \text { then }(f x, f y) \in E(G),
$$

2. There exists $a \in\left[0, \frac{1}{2}\right)$ such that

$$
\sigma_{r}(f x, f y) \leq a\left(\sigma_{r}(x, f x)+\sigma_{r}(y, f y)\right) \forall x, y \in E(G) .
$$

Remark 2. If $f$ is a $G_{r}$-Kannan mapping, then $f$ is both a $G_{r}^{-1}$-Kannan mapping and $\tilde{G}_{r}$-Kannan mapping.

Lemma 1. Let $\left(X, \sigma_{r}\right)$ be a rectangular metric-like space endowed with a graph $G$ and $f: X \longrightarrow X$ be $G_{r}$-Kannan mapping with constant $a$. If the graph $G$ is weakly connected, then, given $x, y \in X$, there exists $r(x, y) \geq 0$ such that

$$
\sigma_{r}\left(f^{n} x, f^{n} y\right) \leq a \sigma_{r}\left(f^{n-1} x, f^{n} x\right)+\left(\frac{a}{1-a}\right)^{n} r(x, y)+a \sigma_{r}\left(f^{n-1} y, f^{n} y\right) \forall n \in \mathbb{N} .
$$

Proof. For every $x, y \in X$, we need to consider the following two cases.

If $(x, y) \in \tilde{G}$, then, by induction, $\left(f^{n} x, f^{n} y\right) \in \tilde{G}$; therefore, Label (5) is true by taking $r(x, y)=0$ for all $n \in \mathbb{N}$.

If $(x, y) \notin \tilde{G}$, then there is a path $\left(x_{i}\right)_{i=0}^{N}$ in $\tilde{G}$ from $x$ to $y$ such that $x_{0}=x, \ldots, x_{N}=y$ with $\left(x_{i-1}, x_{i}\right) \in E(\tilde{G})$ for $i=1, \ldots, N$ and $\left(x_{i}, f x_{i}\right) \in E(\tilde{G})$ for $i=1, \ldots, N-1$.

Since $f$ is a $\tilde{G}_{r}$-Kannan contractive, then, by induction in Label (3), we get

$$
\left(f^{n} x_{i-1}, f^{n} x_{i}\right) \in E(\tilde{G}) .
$$

Now, by using the second property of $G_{r}$-Kannan contraction given by (4), we obtain

$$
\sigma_{r}\left(f^{2} x_{i}, f x_{i}\right) \leq a\left[\sigma_{r}\left(f x_{i}, f^{2} x_{i}\right)+\sigma_{r}\left(x_{i}, f x_{i}\right)\right] .
$$


By induction, we obtain

$$
\sigma_{r}\left(f^{n-1} x_{i}, f^{n} x_{i}\right) \leq\left(\frac{a}{1-a}\right)^{n-1} \sigma_{r}\left(x_{i}, f x_{i}\right),
$$

knowing that the considered graph $G$ is simple and from (6) we can conclude that $f^{n-1} x_{i} \neq f^{n} x_{i}$ for all $n \in \mathbb{N}$, which allow us to use the rectangular inequality below.

Now, in order to use the rectangular inequality, we need to consider the cases $N$ odd and $N$ even:

Case 1: $N=2 p+1$ (i.e., $N$ odd)

$$
\begin{aligned}
\sigma_{r}\left(f^{n} x, f^{n} y\right) & =\sigma_{r}\left(f^{n} x_{0}, f^{n} x_{N}\right) \\
& \leq \sigma_{r}\left(f^{n} x_{0}, f^{n} x_{1}\right)+\sigma_{r}\left(f^{n} x_{1}, f^{n} x_{2}\right)+\sigma_{r}\left(f^{n} x_{2}, f^{n} x_{3}\right)+\ldots \sigma_{r}\left(f^{n} x_{N-1}, f^{n} x_{N}\right) \\
& \leq a\left[\sigma_{r}\left(f^{n-1} x_{0}, f^{n} x_{0}\right)+\sigma_{r}\left(f^{n-1} x_{1}, f^{n} x_{1}\right)+\sigma_{r}\left(f^{n-1} x_{1}, f^{n} x_{1}\right)\right. \\
& \left.+\sigma_{r}\left(f^{n-1} x_{2}, f^{n} x_{2}\right)+\ldots+\sigma_{r}\left(f^{n-1} x_{N-1}, f^{n} x_{N-1}\right)+\sigma_{r}\left(f^{n-1} x_{N}, f^{n} x_{N}\right)\right] \\
& \leq a\left[\sigma_{r}\left(f^{n-1} x_{0}, f^{n} x_{0}\right)+2 \sum_{k=1}^{N-1} \sigma_{r}\left(f^{n-1} x_{k}, f^{n} x_{k}\right)+\sigma_{r}\left(f^{n-1} x_{N}, f^{n} x_{N}\right)\right] .
\end{aligned}
$$

Using (7), we get

$$
\sigma_{r}\left(f^{n} x, f^{n} y\right) \leq a \sigma_{r}\left(f^{n-1} x, f^{n} x\right)+\left(\frac{a}{1-a}\right)^{N} r(x, y)+a \sigma_{r}\left(f^{n-1} y, f^{n} y\right),
$$

where $r(x, y)=2(1-a) \sum_{k=1}^{N-1} \sigma_{r}\left(x_{k}, f x_{k}\right)$.

Case 2: $N=2 p$ (i.e., $N$ even)

$$
\begin{aligned}
\sigma_{r}\left(f^{n} x, f^{n} y\right) & =\sigma_{r}\left(f^{n} x_{0}, f^{n} x_{N}\right) \\
& \leq \sigma_{r}\left(f^{n} x_{0}, f^{n} x_{1}\right)+\sigma_{r}\left(f^{n} x_{1}, f^{n} x_{2}\right)+\ldots+\sigma_{r}\left(f^{n} x_{N-3}, f^{n} x_{N-2}\right)+\sigma_{r}\left(f^{n} x_{N-2}, f^{n} x_{N}\right) \\
& \leq a\left[\sigma_{r}\left(f^{n-1} x_{0}, f^{n} x_{0}\right)+\sigma_{r}\left(f^{n-1} x_{1}, f^{n} x_{1}\right)+\sigma_{r}\left(f^{n-1} x_{1}, f^{n} x_{1}\right)\right. \\
& \left.+\sigma_{r}\left(f^{n-1} x_{N-2}, f^{n} x_{N-2}\right)+\sigma_{r}\left(f^{n-1} x_{N-2}, f^{n} x_{N}\right)+\sigma_{r}\left(f^{n-1} x_{N}, f^{n} x_{N}\right)\right] \\
& \leq a\left[\sigma_{r}\left(f^{n-1} x_{0}, f^{n} x_{0}\right)+2\left(\frac{a}{1-a}\right)^{n-1} \sum_{k=1}^{N-2} \sigma_{r}\left(f^{n-1} x_{k}, f^{n} x_{k}\right)+\sigma_{r}\left(f^{n-1} x_{N}, f^{n} x_{N}\right)\right] \\
& \leq a \sigma_{r}\left(f^{n-1} x, f^{n} x\right)+\left(\frac{a}{1-a}\right)^{n} r(x, y)+a \sigma_{r}\left(f^{n-1} y, f^{n} y\right),
\end{aligned}
$$

where $r(x, y)=2(1-a) \sum_{k=1}^{N-2} \sigma_{r}\left(x_{k}, f x_{k}\right)$.

Finally, we obtain

$$
\sigma_{r}\left(f^{n} x, f^{n} y\right) \leq a \sigma_{r}\left(f^{n-1} x, f^{n} x\right)+\left(\frac{a}{1-a}\right)^{n} r(x, y)+a \sigma_{r}\left(f^{n-1} y, f^{n} y\right),
$$

where

$$
r(x, y)= \begin{cases}2(1-a) \sum_{k=1}^{N-1} \sigma_{r}\left(x_{k}, f x_{k}\right), & \text { if } N=2 p+1 \\ 2(1-a) \sum_{k=1}^{N-2} \sigma_{r}\left(x_{k}, f x_{k}\right), & \text { if } N=2 p .\end{cases}
$$


Theorem 1. Let $\left(X, \sigma_{r}\right)$ be a $\sigma_{r}$-complete rectangular metric-like space endowed with a graph $G$ and $f: X \longrightarrow$ $X$ be a continuous $G_{r}-K a n n a n$ contraction. We suppose that:

1. $G$ is weakly connected,

2. for any $\left(x_{n}\right)_{n \in \mathbb{N}}$, if $x_{n} \longrightarrow x$ as $n \longrightarrow \infty$ and $\left(x_{n}, x_{n+1}\right) \in E(G) \forall n \in \mathbb{N}$, then there is a subsequence $\left(x_{k_{n}}\right)_{n \in \mathbb{N}}$ such that $\left(x_{k_{n}}, x\right) \in E(G) \forall n \in \mathbb{N}$.

Then, $f$ has a unique fixed point and $\lim _{n \rightarrow \infty} f^{n} x=x^{*}$.

Proof. Let $x \in X$. By taking $y=f x$ in lemma 1 , we obtain

$$
\sigma_{r}\left(f^{n} x, f^{n+1} x\right) \leq a \sigma_{r}\left(f^{n-1} x, f^{n} x\right)+\left(\frac{a}{1-a}\right)^{n} r(x, f x)+a \sigma_{r}\left(f^{n} y, f^{n+1} x\right) \forall n \in \mathbb{N}^{*} .
$$

Therefore,

$$
\sigma_{r}\left(f^{n} x, f^{n+1} x\right) \leq \frac{a}{1-a} \sigma_{r}\left(f^{n-1} x, f^{n} x\right)+\frac{a^{n}}{(1-a)^{n+1}} r(x, f x) .
$$

Now, using (7), we get

$$
\sigma_{r}\left(f^{n} x, f^{n+1} x\right) \leq\left(\frac{a}{1-a}\right)^{n} \sigma_{r}(x, f x)+n \frac{a^{n}}{(1-a)^{n+1}} r(x, f x) .
$$

Let $\alpha=\frac{a}{1-a}$; then, $\alpha \in[0,1)$. From (8), we have

$$
\begin{aligned}
\sum_{k=0}^{n} \sigma_{r}\left(f^{k} x, f^{k+1} x\right) & \leq \sum_{k=0}^{n} \alpha^{k} \sigma_{r}(x, f x)+\frac{1}{1-a} \sum_{k=0}^{n} r(x, f x) k \alpha^{k} \\
& =\sigma_{r}(x, f x) \frac{1-\alpha^{n+1}}{1-\alpha}+\frac{r(x, f x)}{1-a} \sum_{k=0}^{n} k \alpha^{k}
\end{aligned}
$$

Since $\alpha<1$, and the sequence $\sum_{k=0}^{\infty} k \alpha^{k}$ is equivalent to $o\left(\frac{1}{n^{2}}\right)$ that is convergent. Then,

$$
\sum_{k=0}^{\infty} \sigma_{r}\left(f^{k} x, f^{k+1} x\right)<\infty
$$

Let's prove that $\left(f^{n} x\right)_{n \geq 0}$ is a $\sigma_{r}$-Cauchy sequence; that is, $\lim _{n, p \rightarrow \infty} \sigma_{r}\left(f^{n} x, f^{n+p} x\right)=0$ for all $n, p \in \mathbb{N}$.

To use the rectangular inequality, we need to consider the following two cases:

Case 1: $p=2 s+1$

$$
\begin{aligned}
\sigma_{r}\left(f^{n} x, f^{n+p} x\right) & \leq \sigma_{r}\left(f^{n} x, f^{n+1} x\right)+\sigma_{r}\left(f^{n+1} x, f^{n+2} x\right)+\ldots \sigma_{r}\left(f^{n+p-1} x, f^{n+p} x\right) \\
& \leq \sum_{k=n}^{n+p-1} \sigma_{r}\left(f^{k} x, f^{k+1} x\right) \\
& =\sum_{k=0}^{n+p-1} \sigma_{r}\left(f^{k} x, f^{k+1} x\right)-\sum_{k=0}^{n-1} \sigma_{r}\left(f^{k} x, f^{k+1} x\right) .
\end{aligned}
$$

Thus, from (9), we obtain

$$
\lim _{n \rightarrow \infty} \sigma_{r}\left(f^{n} x, f^{n+p} x\right)=0 \text { for all odd } p \in \mathbb{N} .
$$


Case 2: $p=2 s$

$$
\begin{aligned}
\sigma_{r}\left(f^{n} x, f^{n+p} x\right) & \leq \sigma_{r}\left(f^{n} x, f^{n+1} x\right)+\sigma_{r}\left(f^{n+1} x, f^{n+2} x\right)+\ldots+\sigma_{r}\left(f^{n+p-2} x, f^{n+p} x\right) \\
& \leq \sum_{k=n}^{n+p-1} \sigma_{r}\left(f^{k} x, f^{k+1} x\right)+\sigma_{r}\left(f^{n+p-2} x, f^{n+p} x\right) \\
& =\sum_{k=0}^{n+p-1} \sigma_{r}\left(f^{k} x, f^{k+1} x\right)-\sum_{k=0}^{n-1} \sigma_{r}\left(f^{k} x, f^{k+1} x\right)+\sigma_{r}\left(f^{n+p-2} x, f^{n+p} x\right) .
\end{aligned}
$$

Similarly, in case 1 , we have $\sum_{k=0}^{n+p-1} \sigma_{r}\left(f^{k} x, f^{k+1} x\right)-\sum_{k=0}^{n-1} \sigma_{r}\left(f^{k} x, f^{k+1} x\right) \longrightarrow 0$ as $n \longrightarrow \infty$. Let's prove that $\sigma_{r}\left(f^{n+p-2} x, f^{n+p} x\right) \longrightarrow 0$.

Using the second condition of the $G_{r}$-Kannan contraction, we obtain

$$
\begin{aligned}
\sigma_{r}\left(f^{n+p-2} x, f^{n+p} x\right) & \leq a\left[\sigma_{r}\left(f^{n+p-3} x, f^{n+p-2} x\right)+\sigma_{r}\left(f^{n+p-1} x, f^{n+p} x\right)\right] \\
& \leq a \sum_{k=0}^{n+p} \sigma_{r}\left(f^{k} x, f^{k+1} x\right) \longrightarrow 0 \text { as } n \longrightarrow \infty
\end{aligned}
$$

Then,

$$
\sigma_{r}\left(f^{n} x, f^{n+p} x\right) \longrightarrow 0 \text { as } n \longrightarrow \infty \text { for all even } p \in \mathbb{N}
$$

Therefore, from (11) and (13), we obtain that $\sigma_{r}\left(f^{n} x, f^{n+p} x\right)$ converges to 0 as $n, p \longrightarrow \infty$. Thus, $\left(f^{n} x\right)_{n \geq 0}$ is a $\sigma_{r}$-Cauchy sequence. From the completeness of $(X, \sigma)$, there exists some $x^{*} \in X$ such that $\lim _{n \rightarrow \infty} f^{n} x=x^{*}$.

Now, let's prove that $x^{*}$ is a fixed point of $f$. Since the graph $G$ is weakly connected, there is at least $x_{0} \in X$ such that $\left(x_{0}, f x_{0}\right) \in E(\tilde{G})$; then, $\left(f^{n} x_{0}, f^{n+1} x_{0}\right) \in E(\tilde{G}) \forall n \in \mathbb{N}$.

Using the second assertion of the theorem and given the fact that $\lim _{n \rightarrow \infty} f^{n} x_{0}=x^{*}$, there is a subsequence $\left(f^{k_{n}} x_{0}\right)_{n \geq 0}$ such that $\left(f^{k_{n}} x_{0}, f x^{*}\right) \in E(G)$ for all $n \in \mathbb{N}$.

Now, let's apply the rectangular inequality to $\sigma_{r}\left(x^{*}, f x^{*}\right)$. Due to the Definition 2, we need to consider the following cases:

Case 1: If $x^{*} \neq f^{k_{n}+1} x_{0}, x^{*} \neq f^{k_{n}+2} x_{0}, f x^{*} \neq f^{k_{n}+2} x_{0}$ and $f x^{*} \neq f^{k_{n}+1} x_{0}$,

we obtain

$$
\begin{aligned}
\sigma_{r}\left(x^{*}, f x^{*}\right) & \leq \sigma_{r}\left(x^{*}, f^{k_{n}+1} x_{0}^{*}\right)+\sigma_{r}\left(f^{k_{n}+1} x_{0}^{*}, f^{k_{n}+2} x_{0}^{*}\right)+\sigma_{r}\left(f^{k_{n}+2} x_{0}^{*}, f x^{*}\right) \\
& \leq \sigma_{r}\left(x^{*}, f^{k_{n}+1} x_{0}^{*}\right)+\sigma_{r}\left(f^{k_{n}+1} x_{0}^{*}, f^{k_{n}+2} x_{0}^{*}\right)+a\left[\sigma_{r}\left(f^{k_{n}+1} x_{0}^{*}, f^{k_{n}+2} x^{*}\right)+\sigma_{r}\left(x^{*}, f x^{*}\right)\right] .
\end{aligned}
$$

Then,

$$
\sigma_{r}\left(x^{*}, f x^{*}\right) \leq \frac{1}{1-a} \sigma_{r}\left(x^{*}, f^{k_{n}+1} x_{0}^{*}\right)+\frac{2}{1-a} \sigma_{r}\left(f^{k_{n}+1} x_{0}^{*}, f^{k_{n}+2} x_{0}^{*}\right) .
$$

Using the fact that $\lim _{n \rightarrow \infty} f^{n} x=x^{*}$, we get

$$
\begin{aligned}
\sigma_{r}\left(x^{*}, f x^{*}\right) & \leq \frac{1}{1-a} \sigma_{r}\left(x^{*}, x^{*}\right)+\frac{2}{1-a} \sigma_{r}\left(x^{*}, x^{*}\right) \\
& =\frac{3}{1-a} \sigma_{r}\left(x^{*}, x^{*}\right) .
\end{aligned}
$$

Since in rectangular metric-like space $\sigma_{r}\left(x^{*}, x^{*}\right)$ is not necessarily zero, we need to prove it.

Let $x \in X$, by taking $y=x$ in Lemma 1 , we obtain:

$$
\sigma_{r}\left(f^{n} x, f^{n} x\right) \leq a \sigma_{r}\left(f^{n-1} x, f^{n} x\right)+\left(\frac{a}{1-a}\right)^{n} r(x, x)+a \sigma_{r}\left(f^{n-1} x, f^{n} x\right) .
$$


Letting $n \longrightarrow \infty$ and knowing that $\lim _{n \rightarrow \infty} f^{n} x=x^{*}$ and $\left(\frac{a}{1-a}\right)^{n} \longrightarrow 0$, we get

$$
\sigma_{r}\left(x^{*}, x^{*}\right) \leq a \sigma_{r}\left(x^{*}, x^{*}\right)+\operatorname{Or}(x, x)+a \sigma_{r}\left(x^{*}, x^{*}\right) .
$$

Since $r(x, x)$ is constant, then $\sigma_{r}\left(x^{*}, x^{*}\right) \leq 2 a \sigma_{r}\left(x^{*}, x^{*}\right)$. Therefore,

$$
\sigma_{r}\left(x^{*}, x^{*}\right)=0 .
$$

Finally, from (15) and (16), we get $\sigma_{r}\left(x^{*}, f x^{*}\right)=0$; then, $f x^{*}=x^{*}$ that is $x^{*}$ is a fixed point of $f$. Case 2: If $x^{*}=f^{k_{n}+1} x_{0}$ and then $f x^{*}=f^{k_{n}+2} x_{0}$,

from (4), we obtain

$$
\begin{aligned}
\sigma_{r}\left(x^{*}, f x^{*}\right) & =\sigma_{r}\left(f^{k_{n}+1} x_{0}, f^{k_{n}+2} x_{0}\right) \\
& \leq a\left[\sigma_{r}\left(f^{k_{n}} x_{0}, f^{k_{n}+1} x_{0}\right)+\sigma_{r}\left(f^{k_{n}+1} x_{0}, f^{k_{n}+2} x_{0}\right)\right] .
\end{aligned}
$$

Then,

$$
\sigma_{r}\left(f^{k_{n}+1} x_{0}, f^{k_{n}+2} x_{0}\right) \leq \frac{a}{1-a} \sigma_{r}\left(f^{k_{n}} x_{0}, f^{k_{n}+1} x_{0}\right) .
$$

Letting $n \longrightarrow \infty$ in (17) and knowing that $\lim _{n \rightarrow \infty} f^{n} x_{0}=x^{*}$, we get

$$
\sigma_{r}\left(x^{*}, f x^{*}\right) \leq \sigma_{r}\left(f^{k_{n}+1} x_{0}, f^{k_{n}+2} x_{0}\right)<\sigma_{r}\left(x^{*}, x^{*}\right) .
$$

Therefore, similarly to the precedent case, we obtain from (16) that $f x^{*}=x^{*}$.

Case 3: If $x^{*}=f^{k_{n}+2} x_{0}$

it is similar to Case 2 .

Case 4: If $f x^{*}=f^{k_{n}+2} x_{0}$

since $\lim _{n \rightarrow \infty} f^{n} x_{0}=x^{*}$, then there exists $N \in \mathbb{N}$ such that $x^{*}=f^{N} x_{0}$ :

$$
\begin{aligned}
\sigma_{r}\left(x^{*}, f x^{*}\right) & =\sigma_{r}\left(f^{N} x_{0}, f^{k_{n}+2} x_{0}\right) \\
& \leq a\left[\sigma_{r}\left(f^{N-1} x_{0}, f^{N} x_{0}\right)+\sigma_{r}\left(f^{k_{n}+1} x_{0}, f^{k_{n}+2} x_{0}\right)\right] \\
& <\sigma_{r}\left(f^{N-1} x_{0}, f^{N} x_{0}\right)+\sigma_{r}\left(f^{k_{n}+1} x_{0}, f^{k_{n}+2} x_{0}\right) .
\end{aligned}
$$

Letting $n \longrightarrow \infty$ in (20), we get

$$
\sigma_{r}\left(f^{N-1} x_{0}, f^{N} x_{0}\right)+\sigma_{r}\left(f^{k_{n}+1} x_{0}, f^{k_{n}+2} x_{0}\right) \longrightarrow 2 \sigma_{r}\left(x^{*}, x^{*}\right) \text { as } n \longrightarrow \infty .
$$

Therefore, $\sigma_{r}\left(x^{*}, f x^{*}\right)<2 \sigma_{r}\left(x^{*}, x^{*}\right)$ and, from (16), we obtain $f x^{*}=x^{*}$.

Case 5: If $f x^{*} \neq f^{k_{n}+2} x_{0}$ and $x^{*}=f^{k_{n}+1} x_{0}$, this leads to a contradiction

Case 6: If $f x^{*} \neq f^{k_{n}+1} x_{0}$ and $x^{*}=f^{k_{n}+2} x_{0}$, it is the same reasoning in Case 5.

Let's prove the uniqueness of $x^{*}$. Consider two fixed points $x^{*}$ and $y^{*}$ in $X$ such that $x^{*}=\lim _{n \rightarrow \infty} f^{n} x$ and $y^{*}=\lim _{n \rightarrow \infty} f^{n} y$. By letting $n \longrightarrow \infty$ in (5) and using (16), we obtain that $\sigma_{r}\left(x^{*}, y^{*}\right) \leq 0$; therefore, $x^{*}=y^{*}$. 
Example 3. Let $X=[0,1]$ be endowed with the following rectangular metric-like

$$
\sigma_{r}\left(x_{1}, x_{2}\right)=\left\{\begin{array}{cl}
1, & \text { if } x_{1}=x_{2} \\
\frac{x_{1}+x_{2}}{2}, & \text { otherwise. }
\end{array}\right.
$$
$f x=\frac{x}{2}$.

Define the graph $G$ by $E(G)=\left\{\left(x_{1}, x_{2}\right) \in[0,1] \times[0,1]: x_{1} \geq x_{2}\right\}$ and let $f$ self-map on $[0,1]$ such that

It is easy to verify that $\left(X, \sigma_{r}\right)$ is a $\sigma_{r}$-complete rectangular metric-like space, $G$ is weakly connected and $f$ is $a G_{r}$-Kannan contraction with $a=\frac{2}{5}$. Then, by Theorem $1, f$ has a unique fixed point that is $u=0$.

Next, we prove the existence of a fixed point under another G-contraction called G-graphic contraction.

Definition 9. Let $\left(X, \sigma_{r}\right)$ be a rectangular metric-like space endowed with a graph $G$. The mapping $f: X \longrightarrow$ $X$ is a G-graphic contraction if the following conditions hold:

- $\quad f$ preserve edge of $G:(x, y) \in E(G) \Longrightarrow(f x, f y) \in E(G) \forall x, y \in X$,

- $\quad$ there exists $\alpha \in[0,1)$ such that

$$
\begin{aligned}
& \sigma_{r}\left(f x, f^{2} x\right) \leq \alpha \sigma_{r}(x, f x) \forall x \in X^{f}, \\
& \sigma_{r}\left(f x, f^{3} x\right) \leq \alpha \sigma_{r}\left(x, f^{2} x\right) \forall x \in X^{f},
\end{aligned}
$$

where $X^{f}=\{x \in X:(x, f x) \in E(G)$ or $(f x, x) \in E(G)\}$.

Definition 10. A mapping $f: X \longrightarrow X$ is called orbitally $G$-continuous if for all $a, b \in X$ and any sequence $\left(k_{n}\right)_{n \in \mathbb{N}}$,

$$
\left(f^{k_{n}} a\right) \text { convergents to } b \text { and }\left(f^{k_{n}} a, f^{k_{n}+1} a\right) \in E(G) \forall n \in \mathbb{N} \text { implies } f\left(f^{k_{n}} a\right) \longrightarrow f b \text {. }
$$

Lemma 2. Let $\left(X, \sigma_{r}\right)$ be a rectangular metric-like space endowed with a graph $G$. Let $f: X \longrightarrow X$ be a $G$-graphic contraction. If $x \in X^{f}$, then there exists $r(x) \geq 0, r_{1}(x) \geq 0$ such that

$$
\begin{aligned}
& \sigma_{r}\left(f^{n}, f^{n+1} x\right) \leq \alpha^{n} r(x) \forall n \in \mathbb{N}, \\
& \sigma_{r}\left(f^{n}, f^{n+2} x\right) \leq \beta^{n} r_{1}(x) \forall n \in \mathbb{N} .
\end{aligned}
$$

Proof. Consider $x \in X^{f}$, and let's discuss the two cases $(x, f x) \in E(G)$ and $(f x, x) \in E(G)$.

If $(x, f x) \in E(G)$, then, by induction, we obtain $\left(f^{n} x, f^{n+1} x\right) \in E(G)$. Thus, using (20), we get

$$
\sigma_{r}\left(f^{n}, f^{n+1} x\right) \leq \alpha^{n} r(x),
$$

where $r(x)=\sigma_{r}(x, f x)$. If $(f x, x) \in E(G)$, we have $\left(f^{n+1} x, f^{n} x\right) \in E(G)$. By the same procedure above, we obtain the desired result.

Similarly, we prove the inequality (23) where $r_{1}(x)=\sigma_{r}\left(x, f^{2} x\right)$.

Theorem 2. Let $\left(X, \sigma_{r}\right)$ be a $\sigma_{r}$-complete rectangular metric-like space endowed with a graph $G$. Let $f: X \longrightarrow$ $X$ be a G-graphic contraction and orbitally G-continuous.

Suppose that the triplet $\left(X, \sigma_{r}, G\right)$ satisfies the following condition $(P)$ :

For any $\left(x_{n}\right)_{n \in \mathbb{N}}$ in $X$, if $x_{n} \longrightarrow x$ and $\left(x_{n}, x_{n+1}\right) \in E(G)$ (or respectively $\left.\left(x_{n+1}, x_{n}\right) \in E(G)\right) \forall n \in \mathbb{N}$, then there is a subsequence $\left(x_{k_{n}}\right)_{n \in \mathbb{N}}$ with $\left(x_{k_{n}}, x\right) \in E(G)$ (or respectively $\left(x, x_{k_{n}}\right) \in E(G)$ ). 
Then, the following statements hold:

1. For any $x \in X^{f}$, the restriction of $f$ to $[x]_{\tilde{G}}$ has a fixed point.

2. If $G$ is weakly connected and $X^{f} \neq \varnothing, f$ has a fixed point.

Proof. 1. Let $x \in X^{f}$. Then, from lemma 2, there exists $r(x) \geq 0$ such that $\sigma_{r}\left(f^{n} x, f^{n+1}\right) \leq \alpha^{n} r(x)$ $\forall n \in \mathbb{N}$.

Let $n, m \in \mathbb{N} m>n$; we claim that $\sigma_{r}\left(f^{n} x, f^{n+m} x\right) \longrightarrow 0$ as $n, m \longrightarrow \infty$. To prove the claim, we need to consider the following cases.

Case $1:$ If there exists $n \in \mathbb{N}$ such that $f^{n} x=f^{n+1} x$. Then, $f^{n+m} x=f^{n+1} x$ and $\sigma_{r}\left(f^{n} x, f^{n+m} x\right)=\sigma_{r}\left(f^{n} x, f^{n+1} x\right) \leq \alpha^{n} r(x) \longrightarrow 0$ as $n \longrightarrow \infty$.

Case $2: n+m=2 p+1(n+m$ is odd $)$ and for all $n \in \mathbb{N} f^{n} x \neq f^{n+1} x$.

$$
\begin{aligned}
\sigma_{r}\left(f^{n} x, f^{n+m} x\right) & \leq \sigma_{r}\left(f^{n} x, f^{n+1} x\right)+\sigma_{r}\left(f^{n+1} x, f^{n+2} x\right)+\ldots+\sigma_{r}\left(f^{2 p-1} x, f^{2 p} x\right) \\
& +\sigma_{r}\left(f^{2 p-1} x, f^{2 p+1} x\right) \\
& \leq \alpha^{n} r(x)+\alpha^{n+1} r(x)+\ldots+\alpha^{n+m-1} r(x) \\
& \leq \sum_{k=1}^{m} \alpha^{n+k-1} r(x) .
\end{aligned}
$$

Case $3: n+m=2 p(n+m$ is even $)$ and $f^{n} x \neq f^{n+1} x \forall n \in \mathbb{N}$.

$$
\begin{aligned}
\sigma_{r}\left(f^{n} x, f^{n+m} x\right) & \leq \sigma_{r}\left(f^{n} x, f^{n+1} x\right)+\sigma_{r}\left(f^{n+1} x, f^{n+2} x\right)+\ldots+\sigma_{r}\left(f^{n+m-3} x, f^{n+m-2} x\right) \\
& +\sigma_{r}\left(f^{n+m-2} x, f^{n+m} x\right) \\
& \leq \alpha^{n} r(x)+\alpha^{n+1} r(x)+\ldots+\alpha^{n+m-3} r(x)+\sigma_{r}\left(f^{n+m-2} x, f^{n+m} x\right) \\
& \leq \sum_{k=1}^{m-2} \alpha^{n+k-1} r(x)+\sigma_{r}\left(f^{n+m-2} x, f^{n+m} x\right) \\
& \leq \sum_{k=1}^{m+1} \alpha^{n+k-1} r(x)+\sigma_{r}\left(f^{n+m-1} x, f^{n+m+1} x\right) \\
& \leq \sum_{k=1}^{m+1} \alpha^{n+k-1} r(x)+\sigma_{r}\left(f^{n+m-1} x, f^{n+m} x\right)+\sigma_{r}\left(f^{n+m} x, f^{n+m+2} x\right) \\
& +\sigma_{r}\left(f^{n+m+2} x, f^{n+m+1} x\right) \\
& \leq \sum_{k=1}^{m+1} \alpha^{n+k-1} r(x)+\alpha^{n+m-1} r(x)+\alpha^{n+m} r_{1}(x)+\alpha^{n+m+1} r(x) .
\end{aligned}
$$

Using the fact that $0 \leq \alpha<1$ and taking the limits in (24) and (25), we obtain

$$
\sigma_{r}\left(f^{n} x, f^{n+m} x\right) \longrightarrow 0 \text { as } n, m \longrightarrow \infty \text {. }
$$

Hence, $\left(f^{n} x\right)_{n \in \mathbb{N}}$ is a $\sigma_{r}$-Cauchy sequence.

From the $\sigma_{r}$-completeness of the rectangular metric-like space, there exists $u \in X$ such that $f^{n} x \longrightarrow u, \longrightarrow \infty$.

Given that $x \in X^{f}$, we get $f^{n} x \in X^{f}$ for all $n \in \mathbb{N}$.

Now, assume that $(x, f x) \in E(G)$ (which can be done also if $(f x, x) \in E(G)$ ). By the property $(P)$, there exists a subsequence $\left(f^{k_{n}} x\right)_{n \in \mathbb{N}}$ of $\left(f^{n} x\right)_{n \in \mathbb{N}}$ such that $\left(f^{k_{n}} x, u\right) \in E(G) \forall n \in \mathbb{N}$. Thereby, a path in $G$ from $x$ to $u$ can be formed by the points: $x, f x, \ldots, f^{k_{1}} x, u$; then, $u \in[x]_{\tilde{G}}$. 
Since $f$ is orbitally $G$-continuous, we obtain that $f\left(f^{k_{n}} x\right) \longrightarrow f u$, which gives $f u=u$. Thus, $u$ is a fixed point for $f_{[x]_{\tilde{G}}}$.

2. Let $x \in X^{f}$. Since $G$ is weakly connected, we have $X=[x]_{\tilde{G}}$ and, using 1 , we get that $f$ has a fixed point.

Example 4. Let $X=[0,1]$ be endowed with the following rectangular metric-like

$$
\sigma_{r}(a, b)=\left\{\begin{array}{cc}
1, & \text { if } a=b, \\
\frac{a+b}{2}, & \text { otherwise. }
\end{array}\right.
$$

Consider $E(G)=\{(0,0)\} \cup\left\{(0, a), a \geq \frac{1}{2}\right\} \cup\{(a, b), a, b \in(0,1)\}$, and the mapping

$$
f:[0,1] \longrightarrow[0,1] \text { as follows }: f x=\left\{\begin{array}{lc}
\frac{x}{2}, & \text { if } x \in(0,1), \\
0, & \text { if } x=0 \\
1, & \text { if } x=1 .
\end{array}\right.
$$

Then, $G$ is weakly connected and $f$ is a G-graphic contraction. Indeed, $f$ preserves edges of $G$ and satisfies (20). In addition to this, $f$ is orbitally G-continuous. Thereby, all conditions of theorem 2 are satisfied; then, the mapping $f$ has two fixed points that is $f(0)=0$ and $f(1)=1$.

Inspired by the work of [15], we introduce the notion of $(G-\phi)$-contraction and we state a new fixed point theorem.

Definition 11. Let $\left(X, \sigma_{r}\right)$ be a rectangular metric-like space endowed with a graph $G$. A map $f$ is called to be $a(G-\phi)$-contraction if

1. for all $x, y \in X$,

$$
(x, y) \in E(G) \text { implies }(f x, f y) \in E(G)
$$

2. for all $(x, y) \in E(G)$,

$$
\sigma_{r}(f x, f y) \leq \sigma_{r}(x, y)-\phi\left(\sigma_{r}(x, y)\right)
$$

where $\phi:[0, \infty) \longrightarrow[0, \infty)$ is a nondecreasing continuous function such that $\phi(t)=0$ if and only if $t=0$.

Lemma 3. Let $\left(X, \sigma_{r}\right)$ be a rectangular metric-like space and $f: X \longrightarrow X a(G-\phi)$-contractive mapping. Then, for any $x \in X$ and $y \in[x]_{\tilde{G}}$, we have

$$
\lim _{n \rightarrow \infty} \sigma_{r}\left(f^{n} x, f^{n} y\right)=\lim _{n \rightarrow \infty} r\left(f^{n} x, f^{n} y\right)=0,
$$

where $r(x, y)=\sum_{i=1}^{m} \sigma_{r}\left(s_{i-1}, s_{i}\right),\left\{s_{i}\right\}_{i=0}^{m}$ is a path from $x$ to $y$.

Proof. Consider $x \in X$ and $y \in[x]_{\tilde{G}}$. Then, there exists a path $\left\{s_{i}\right\}_{i=0}^{k}$ from $x$ to $y$ in the graph $G$. From (27) and (28), we get $\left(f^{n} s_{i-1}, f^{n} s_{i}\right) \in E(G)$ for all $i=1,2, \ldots, k$ and $n \in \mathbb{N}$ and

$$
\begin{aligned}
\sigma_{r}\left(f^{n} s_{i-1}, f^{n} s_{i}\right) & \leq \sigma_{r}\left(f^{n-1} s_{i-1}, f^{n-1} s_{i}\right)-\phi\left(\sigma_{r}\left(f^{n-1} s_{i-1}, f^{n-1} s_{i}\right)\right), \\
& \leq \sigma_{r}\left(f^{n-1} s_{i-1}, f^{n-1} s_{i}\right) .
\end{aligned}
$$

Thereby, $\left\{\sigma_{r}\left(f^{n} s_{i-1}, f^{n} s_{i}\right)\right\}$ is a nonincreasing sequence. 
Since $\sigma_{r}\left(f^{n} s_{i-1}, f^{n} s_{i}\right) \geq 0$, then the sequence is bounded by 0 and converges to some $l$. By taking the limit as $n \longrightarrow \infty$ in (30), we get $l \leq l-\phi(l) \leq l \Longrightarrow \phi(l)=0$; then, using the property of the mapping $\phi$, we get $l=0$. Therefore,

$$
\lim _{n \rightarrow \infty} \sigma_{r}\left(f^{n} s_{i-1}, f^{n} s_{i}\right)=0 \forall i=1, \ldots, k \forall n \in \mathbb{N} .
$$

Now, we use the rectangular inequality; for that, we need to distinguish the two cases $k$ odd and $k$ even where $k$ is the length of the path between $x$ and $y$.

Case 1: $k=2 p+1$

$$
\begin{aligned}
\sigma_{r}\left(f^{n} x, f^{n} y\right) & =\sigma_{r}\left(f^{n} s_{0}, f^{n} s_{k}\right)=\sigma_{r}\left(f^{n} s_{0}, f^{n} s_{2 p+1}\right) \\
& \leq \sigma_{r}\left(f^{n} s_{0}, f^{n} s_{1}\right)+\sigma_{r}\left(f^{n} s_{1}, f^{n} s_{2}\right)+\sigma_{r}\left(f^{n} s_{2}, f^{n} s_{2 p+1}\right) \\
& \leq \sigma_{r}\left(f^{n} s_{0}, f^{n} s_{1}\right)+\sigma_{r}\left(f^{n} s_{1}, f^{n} s_{2}\right)+\ldots+\sigma_{r}\left(f^{n} s_{2 p}, f^{n} s_{2 p+1}\right) \\
& =\sum_{i=1}^{k} \sigma_{r}\left(f^{n} s_{i-1}, f^{n} s_{i}\right)=r\left(f^{n} x, f^{n} y\right) .
\end{aligned}
$$

By taking the limit as $n \longrightarrow \infty$ and using (32), we obtain

$$
0 \leq \lim _{n \rightarrow \infty} \sigma_{r}\left(f^{n} x, f^{n} y\right) \leq \lim _{n \rightarrow \infty} r\left(f^{n} x, f^{n} y\right)=0 .
$$

Then, $\lim _{n \rightarrow \infty} \sigma_{r}\left(f^{n} x, f^{n} y\right)=\lim _{n \rightarrow \infty} r\left(f^{n} x, f^{n} y\right)=0$.

Case 2: $k=2 p$

$$
\begin{aligned}
\sigma_{r}\left(f^{n} x, f^{n} y\right) & =\sigma_{r}\left(f^{n} s_{0}, f^{n} s_{k}\right)=\sigma_{r}\left(f^{n} s_{0}, f^{n} s_{2 p}\right) \\
& \leq \sigma_{r}\left(f^{n} s_{0}, f^{n} s_{1}\right)+\sigma_{r}\left(f^{n} s_{1}, f^{n} s_{2}\right)+\sigma_{r}\left(f^{n} s_{2}, f^{n} s_{2 p}\right) \\
& \leq \sigma_{r}\left(f^{n} s_{0}, f^{n} s_{1}\right)+\sigma_{r}\left(f^{n} s_{1}, f^{n} s_{2}\right)+\ldots+\sigma_{r}\left(f^{n} s_{2 p-3}, f^{n} s_{2 p-2}\right)+\sigma_{r}\left(f^{n} s_{2 p-2}, f^{n} s_{2 p}\right) \\
& =\sum_{i=1}^{2 p} \sigma_{r}\left(f^{n} s_{i-1}, f^{n} s_{i}\right)-\sigma_{r}\left(f^{n} s_{2 p-2}, f^{n} s_{2 p-1}\right)-\sigma_{r}\left(f^{n} s_{2 p-1}, f^{n} s_{2 p}\right)+\sigma_{r}\left(f^{n} s_{2 p-2}, f^{n} s_{2 p}\right. \\
& \leq \sum_{i=1}^{2 p} \sigma_{r}\left(f^{n} s_{i-1}, f^{n} s_{i}\right)+\sigma_{r}\left(f^{n} s_{k-2}, f^{n} s_{k}\right) . \\
& =r\left(f^{n} x, f^{n} y\right)+\sigma_{r}\left(f^{n} s_{2 p-2}, f^{n} s_{y}\right) .
\end{aligned}
$$

Similar to the result obtained in (32), we obtain that $\lim _{n \rightarrow \infty} \sigma_{r}\left(f^{n} s_{i-2}, f^{n} s_{i}\right)=0$ for all $i=1, \ldots, k$ and $n \in \mathbb{N}$. Then, $\lim _{n \rightarrow \infty} \sigma_{r}\left(f^{n} s_{2 p-2}, f^{n} s_{y}\right)=\lim _{n \rightarrow \infty} \sigma_{r}\left(f^{n} s_{2 p-2}, f^{n} s_{2 p}\right)=0$. Therefore, from (33), we get

$$
\lim _{n \rightarrow \infty} \sigma_{r}\left(f^{n} x, f^{n} y\right)=\lim _{n \rightarrow \infty} r\left(f^{n} x, f^{n} y\right)=0 .
$$

Theorem 3. Let $\left(X, \sigma_{r}\right)$ be a $\sigma_{r}$-complete rectangular metric-like space endowed with a graph $G$. Let $f: X \longrightarrow$ $X a(G-\phi)$-contractive mapping. Suppose that the following conditions hold:

(i) for any sequence $\left\{f^{n} x\right\}$ in $X$ such that $f^{n} x \longrightarrow y \in X$ with $\left(f^{n+1}, f^{n} x\right) \in E(G)$, there exists a subsequence $\left\{f^{n_{k}} x\right\}$ of $\left\{f^{n} x\right\}$ and $n_{0} \in \mathbb{N}$ such that $\left(y, f^{n_{k}} x\right) \in E(G)$ for all $k \geq n_{0}$.

(ii) There exists some $x_{0} \in X_{f}:=\{x \in X /(x, f x) \in E(G)\}$.

Then, $f_{\mid\left[x_{0}\right]_{\tilde{G}}}$ has a unique fixed point $x^{*} \in\left[x_{0}\right]_{\tilde{G}}$ and $f^{n} y$ converges to $x^{*}$ for all $y \in\left[x_{0}\right]_{\tilde{G}}$.

Proof. Let $x_{0} \in X_{f}$ i.e $\left(f x_{0}, x_{0}\right) \in E(G)$ then $f x_{0} \in\left[x_{0}\right]_{\tilde{G}}$. Let's prove that the sequence $\left\{f^{n} x_{0}\right\}$ is $\sigma_{r}$-Cauchy. 
Using Lemma 3, we obtain $\lim _{n \rightarrow \infty} r\left(f^{n+1} x_{0}, f^{n} x_{0}\right)=0$. Then, for $\epsilon>0$, there exists $n_{0} \in \mathbb{N}$ such that

$$
r\left(f^{n_{0}+1} x_{0}, f^{n} x_{0}\right) \leq \inf \left\{\frac{\epsilon}{2}, \phi\left(\sigma_{r}\left(x_{j}, f^{n} x_{0}\right)\right)\right\},
$$

where the vertex $x_{j} \in X$ is adjacent to $f^{n} x_{0}$ with a direct edge. Since $f x_{0} \in\left[x_{0}\right]_{\tilde{G}}$, for example, there exists a path between $x_{0}$ and $f x_{0}$. By induction, we built a path between $f^{n_{0}} x_{0}$ and $f^{n_{0}+1} x_{0}$ in $\tilde{G}$. Subsequently, we get the existence of at least one vertex $x_{j} \in X$ adjacent to $f^{n} x_{0}$.

Let's denote for any $x \in X$ and any $a \in \mathbb{R}_{+}$the following balls:

$$
\begin{aligned}
& B_{a}(x)=\left\{x^{\prime} \in[x]_{\tilde{G}}: r\left(x, x^{\prime}\right)<a \text { for at least one path in } \tilde{G} \text { from } x \text { to } x^{\prime}\right\}, \\
& \overline{B_{a}(x)}=\left\{x^{\prime} \in[x]_{\tilde{G}}: r\left(x, x^{\prime}\right) \leq a \text { for at least one path in } \tilde{G} \text { from } x \text { to } x^{\prime}\right\} .
\end{aligned}
$$

Now, we claim that $f\left(\overline{B_{\epsilon}\left(f^{n_{0}} x_{0}\right)}\right) \subset \overline{B_{\epsilon}\left(f^{n_{0}} x_{0}\right)}$, which leads us to get $f^{n_{0}+k} x_{0} \in\left[f^{n_{0}} x_{0}\right]_{\tilde{G}}$.

Let $y \in \overline{B_{\epsilon}\left(f^{n_{0}} x_{0}\right)}$. Let $\left\{z_{i}\right\}_{i=0}^{k}$ be a path between $z$ and $f^{n_{0}} x_{0}$ such that $z_{0}=y$ and $z_{k}=f^{n_{0}} x_{0}$. Then, $\left\{f z_{i}\right\}_{i=0}^{k}$ is a path between $f y$ and $f f^{n_{0}} x_{0}$. Therefore, $f y \in\left[f^{n_{0}+1} x_{0}\right]_{\tilde{G}}$. Since we proved above that there exists a path between $f^{n_{0}} x_{0}$ and $f^{n_{0}+1} x_{0}$, then $f y \in\left[f^{n_{0}} x_{0}\right]_{\tilde{G}}$.

Thus, two cases arise:

Case 1: If $0<r\left(y, f^{n_{0}} x_{0}\right) \leq \frac{\epsilon}{2}$

Since $y \in\left[f^{n_{0}} x_{0}\right]_{\tilde{G}}$, there exists a path $\left\{f y, \ldots, f^{n_{0}+1} x_{0}, \ldots, f^{n_{0}} x_{0}\right\}$ between $f y$ and $f^{n_{0}} x_{0}$. Then,

$$
\begin{aligned}
r\left(f y, f^{n_{0}} x_{0}\right) & =r\left(f y, f^{n_{0}+1} x_{0}\right)+r\left(f^{n_{0}+1} x_{0}, f^{n_{0}} x_{0}\right) \\
& =\sum_{i=1}^{k} \sigma_{r}\left(f z_{i-1}, f z_{i}\right)+r\left(f^{n_{0}+1} x_{0}, f^{n_{0}} x_{0}\right) .
\end{aligned}
$$

Using (28), we get

$$
\begin{aligned}
r\left(f y, f^{n_{0}} x_{0}\right) & \leq \sum_{i=1}^{k} \sigma_{r}\left(z_{i-1}, z_{i}\right)-\phi\left(\sigma_{r}\left(z_{i-1}, z_{i}\right)\right)+r\left(f^{n_{0}+1} x_{0}, f^{n_{0}} x_{0}\right) \\
& \leq \sum_{i=1}^{k} \sigma_{r}\left(z_{i-1}, z_{i}\right)+r\left(f^{n_{0}+1} x_{0}, f^{n_{0}} x_{0}\right) \\
& =r\left(y, f^{n_{0}} x_{0}\right)+r\left(f^{n_{0}+1} x_{0}, f^{n_{0}} x_{0}\right) \\
& \leq \frac{\epsilon}{2}+\frac{\epsilon}{2}=\epsilon .
\end{aligned}
$$

Case 2: If $\frac{\epsilon}{2}<r\left(y, f^{n_{0}} x_{0}\right) \leq \epsilon$

Similarly, we have

$$
\begin{aligned}
r\left(f y, f^{n_{0}} x_{0}\right) & \leq \sum_{i=1}^{k} \sigma_{r}\left(z_{i-1}, z_{i}\right)-\phi\left(\sigma_{r}\left(z_{i-1}, z_{i}\right)\right)+r\left(f^{n_{0}+1} x_{0}, f^{n_{0}} x_{0}\right) \\
& \leq \sum_{i=1}^{k} \sigma_{r}\left(z_{i-1}, z_{i}\right)-\phi\left(\sigma_{r}\left(z_{k-1}, z_{k}\right)\right)+r\left(f^{n_{0}+1} x_{0}, f^{n_{0}} x_{0}\right) .
\end{aligned}
$$

Using (34), we get $r\left(f^{n_{0}+1} x_{0}, f^{n_{0}} x_{0}\right) \leq \phi\left(\sigma_{r}\left(x_{j}, f^{n_{0}} x_{0}\right)\right)=\phi\left(\sigma_{r}\left(x_{j}, f^{n_{0}} z_{k}\right)\right)$, where $x_{j}$ is a vertex adjacent to $z_{k}$. Let $x_{j}=z_{k-1}$. Then, we obtain $r\left(f^{n_{0}+1} x_{0}, f^{n_{0}} x_{0}\right) \leq \phi\left(\sigma_{r}\left(z_{k-1}, z_{k}\right)\right.$. Therefore, 


$$
\begin{aligned}
r\left(f y, f n_{0} x_{0}\right) & \leq \sum_{i=1}^{k} \sigma_{r}\left(z_{i-1}, z_{i}\right)-\phi\left(\sigma_{r}\left(z_{k-1}, z_{k}\right)\right)+\phi\left(\sigma_{r}\left(z_{k-1}, z_{k}\right)\right) \\
& \leq r\left(y, f^{n_{0}} x_{0}\right) \\
& \leq \epsilon
\end{aligned}
$$

Thus, we proved that, for $y \in \overline{B_{\epsilon}\left(f^{n_{0}} x_{0}\right)}, r\left(f y, f n_{0} x_{0}\right) \leq \epsilon$ i.e., $f y \in \overline{B_{\epsilon}\left(f^{n_{0}} x_{0}\right)}$, which implies that $f\left(\overline{B_{\epsilon}\left(f^{\left.n_{0} x_{0}\right)}\right)} \subset \overline{B_{\epsilon}\left(f^{\left.n_{0} x_{0}\right)}\right.}\right.$; thereafter, the claim was proved.

Since $f^{n_{0}+1} x_{0} \in \overline{B_{\epsilon}\left(f^{n_{0}} x_{0}\right)}$, then $f^{n_{0}+2} x_{0}=f^{n_{0}+1} x_{0} \subset \overline{B_{\epsilon}\left(f^{n_{0}} x_{0}\right)}$. By repeating this procedure for $k=1,2, \ldots$ and knowing that $f^{n_{0}+k} x_{0} \in\left[f^{n_{0}} x_{0}\right]_{\tilde{G}}$, it follows that $f^{n} x_{0} \in \overline{B_{\epsilon}\left(f^{n_{0}} x_{0}\right)}$ for all $n \geq n_{0}$. Thus,

$$
\sigma_{r}\left(f^{n} x_{0}, f^{n_{0}} x_{0}\right) \leq r\left(f^{n} x_{0}, f^{n_{0}} x_{0}\right) \leq \epsilon,
$$

which gives us that $f^{n} x_{0} \in \overline{B_{\epsilon}\left(f^{n_{0}} x_{0}\right)} \forall n \geq n_{0}$. Finally, $\left\{f^{n} x_{0}\right\}$ is a $\sigma_{r}$-Cauchy sequence in $X$. Since $X$ is $\sigma_{r}$-complete, there exists $l \in X$ such that $f^{n} x_{0} \longrightarrow l, n \longrightarrow \infty$.

From condition $i$, there exists a subsequence $\left\{f^{n_{k}} x_{0}\right\}$ of $\left\{f^{n} x_{0}\right\}$ and $n_{0} \in \mathbb{N}$ such that $\left(l,\left\{f^{n_{k}} x_{0}\right\}\right) \in$ $E(G) \forall k \in \mathbb{N}$ and $k \geq n_{0}$.

Using (28), we get

$$
\begin{aligned}
\sigma_{r}\left(f l, f^{n_{k}+1} x_{0}\right) & \leq \sigma_{r}\left(l, f^{n_{k}} x_{0}\right)+\phi\left(\sigma_{r}\left(l, f^{n_{k}} x_{0}\right)\right) \\
& =\sigma_{r}\left(l, f f^{n_{k}-1} x_{0}\right)+\phi\left(\sigma_{r}\left(l, f f^{n_{k}-1} x_{0}\right)\right) .
\end{aligned}
$$

Since $f^{n_{k}} x_{0} \longrightarrow l$ as $n \longrightarrow \infty$ for all $n \in \mathbb{N}$, then we obtain $\lim _{\rightarrow \infty} f^{n_{k}-1} x_{0}=\lim _{\rightarrow \infty} f^{n_{k}+1} x_{0}=0$ Letting $k \longrightarrow \infty$ in (38), we get

$$
\sigma_{r}(f l, l) \leq \sigma_{r}(l, f l)+\phi\left(\sigma_{r}(l, f l)\right) .
$$

Thus, $\phi\left(\sigma_{r}(l, f l)\right)=0$ and, from the property of $\phi$, we have $\sigma_{r}(l, f l)=0$. Then, $f l=l$; that is, $l$ is a fixed point of $f$.

Let's prove that $f^{n} y \longrightarrow l$, as $n \longrightarrow \infty$. We consider the path $\left\{x_{0}, f x_{0}, \ldots, f^{n_{1}} x_{0}, \ldots, f^{n_{0}} x_{0}, l\right\}$ between $x_{0}$ and $l$ in $\tilde{G}$. Then, $l \in\left[x_{0}\right]_{\tilde{G}}$. Let $y \in\left[x_{0}\right]_{\tilde{G}}$ arbitrary. Then, from Lemma 3, we obtain

$$
\lim _{n \rightarrow \infty} \sigma_{r}\left(f^{n} y, f^{n} x_{0}\right)=0 \Longrightarrow \lim _{n \rightarrow \infty} f^{n} y=l .
$$

Now, suppose that $f$ has two fixed points $u$ and $v$. Then, from Lemma 3, $\sigma_{r}(u, v)=$ $\sigma_{r}\left(f^{n} u, f^{n} v\right) \longrightarrow 0$ as $n \longrightarrow \infty$. Hence, $u=v$ and we get the uniqueness of the fixed point.

Example 5. Consider $X=[0, \infty)$ and the rectangular metric-like $\sigma_{r}(x, y)=\max \{x, y\}$. Then, it is easy to verify that $(X, \sigma)$ is a complete rectangular metric-like space. Let us define the mapping $f$ for each $x \in X$ by $f x=\frac{x^{2}}{x+1}$ and $\phi(t)=\frac{t}{t+1}$.

Let $E(G)=\{(x, y) \in[0, \infty) \times[0, \infty) \mid x \geq y\}$.

We may assume w.l.o.g that $y \leq x$. Then, it is easy to prove that $f$ is a $(G-\phi)$-contraction and satisfies the conditions of Theorem 3. Thus, $f$ has a fixed point $(u=0)$.

\section{Conclusions}

In closing, the author would like to bring to the reader's attention that the following question is still open.

Question 1. Let $\left(X, \sigma_{r}\right)$ be a $\sigma_{r}$-complete rectangular metric-like space endowed with a graph $G$ and $g: X \longrightarrow$ $X$ be a continuous mapping such that 
1.

$$
\forall x, y \in X, \text { If }(x, y) \in E(G), \text { then }(g x, g y) \in E(G),
$$

2. There exists $0 \leq a<\frac{1}{2}$ such that

$$
\sigma_{r}(g x, g y) \leq a \max \left\{\sigma_{r}(x, g x)+\sigma_{r}(y, g y), \sigma_{r}(x, y)\right\} \quad \forall x, y \in E(G) .
$$

In addition, assume that:

1. $G$ is weakly connected.

2. For any $\left(t_{n}\right)_{n \in \mathbb{N}}$, if $\left(t_{n}, t_{n+1}\right) \in E(G) \forall n \in \mathbb{N}$ and $\lim _{n \rightarrow \infty} t_{n}=t$; then, there is a subsequence $\left(t_{k_{n}}\right)_{n \in \mathbb{N}}$ with $\left(t_{k_{n}}, t\right) \in E(G) \forall n \in \mathbb{N}$.

Given all the above conditions, does $g$ have a unique fixed point?

Note that, if the above conjecture is true, then it will have a variety of interesting applications.

Question 2. Let $\left(X, \sigma_{r}\right)$ be a $\sigma_{r}$-complete rectangular metric-like space endowed with a graph $G$ and $g: X \longrightarrow$ $X$ be a continuous mapping such that

1.

$$
\forall x, y \in X, \text { If }(x, y) \in E(G), \text { then }(g x, g y) \in E(G),
$$

2. There exists $0 \leq a<\frac{1}{2}$ such that

$$
\sigma_{r}(g x, g y) \leq a \max \left\{\sigma_{r}(x, y), \frac{\sigma_{r}(x, x)+\sigma_{r}(y, y)}{2}\right\} \forall x, y \in E(G) .
$$

In addition, assume that:

1. $G$ is weakly connected.

2. For any $\left(t_{n}\right)_{n \in \mathbb{N}}$, if $\left(t_{n}, t_{n+1}\right) \in E(G) \forall n \in \mathbb{N}$ and $\lim _{n \rightarrow \infty} t_{n}=t$, then there is a subsequence $\left(t_{k_{n}}\right)_{n \in \mathbb{N}}$ with $\left(t_{k_{n}}, t\right) \in E(G) \forall n \in \mathbb{N}$.

Given all the above conditions, does $g$ have a unique fixed point?

Author Contributions: These authors contributed equally to this work.

Funding: Deanship of Scientific Research, King Saud University: RG-1438-079.

Acknowledgments: This research has been funded by the Deanship of Scientific Research at King Saud University through the Research Group Project No. RG-1438-079.

Conflicts of Interest: The authors declare no conflict of interest.

\section{References}

1. Espinola, R.; Kirk, W.A. Fixed point theorems in R-trees with applications to graph theory. Topol. Its Appl. 2006, 153, 1046-1055. [CrossRef]

2. Jachymski, J. The contraction principle for mappings on a metric space with a graph. Proc. Am. Math. Soc. 2008, 136, 1359-1373. [CrossRef]

3. Altun, I.; Acar, O. Fixed point theorems for weak contractions in the sense of Berinde on partial metric spaces. Topol. Its Appl. 2012, 159, 2642-2648. [CrossRef]

4. Beg, I.; Butt, A.R.; Radojevic, S. The contraction principle for set valued mapings on a metric space with a graph. Comput. Math. Appl. 2010, 60, 1214-1219. [CrossRef]

5. Berinde, V.; Pacurar, M. Fixed points and continuity of almost contractions. Fixed Point Theory 2008, 9, 23-34.

6. Samreen, M.; Kamran, T.; Shahzad, N. Some Fixed Point Theorems in b-Metric Space Endowed with Graph. Abstr. Appl. Anal. 2013, 967132. [CrossRef] 
7. Tripak, O. Common fixed points of G-nonexpansive mappings on Banach spaces with a graph. Fixed Point Theory Appl. 2016, 2016, 87. [CrossRef]

8. Abbas, M.; Ali, B.; Petrusel, G. Fixed points of set-valued contractions in partial metric spaces endowed with a graph. Carpathian J. Math. 2014, 30, 129-137.

9. Samreen, M.; Kamran, T. Fixed Point Theorems for Weakly Contractive Mappings on a Metric Space Endowed with a Graph. Filomat 2014, 441-450. [CrossRef]

10. Aleomraninejad, S.M.A.; Rezapour, S.; Shahzad, N. Fixed point results on subgraphs of directed graphs. Math. Sci. 2013, 7, 41. [CrossRef]

11. Kamran, T.; Postolache, M.; Fahimuddin; Ali, M.U. Fixed point theorems on generalized metric space endowed with graph. J. Nonlinear Sci. Appl. 2016, 9, 4277-4285. [CrossRef]

12. Bejenaru, A.; Pitea, A. Fixed point and best proximity point theorems on partial metric spaces. J. Math. Anal. 2016, 7, 25-44.

13. Shatanawi, W.; Postolache, M. Coincidence and fixed point results for generalized weak contractions in the sense of Berinde on partial metric spaces. Fixed Point Theory Appl. 2013, 2013, 54. [CrossRef]

14. Hui, L.; Jun, Z.; Cheng, L.L. Application Examples of the Network Fixed Point Theory for Space-air-ground Integrated Communication Network. In Proceedings of the International Congress on Ultra Modern Telecommunications and Control Systems and Workshops (ICUMT), Moscow, Russia, 18-20 October 2010.

15. Amini, H. Metric like spaces, partial metric spaces and fixed points. Fixed Point Theory Appl. 2012, $2012,204$. [CrossRef]

16. Mlaiki, N.; Abodayeh, K.; Aydi, H.; Abdeljawad, T.; Abuloha, M. Rectangular metric like type spaces and related fixed points. J. Math. Volume 2018, 2018, 3581768. [CrossRef]

17. Kannan, R. Some results on fixed points-II. Am. Math. Mon. 1969, 76, 405-408.

(C) 2018 by the authors. Licensee MDPI, Basel, Switzerland. This article is an open access article distributed under the terms and conditions of the Creative Commons Attribution (CC BY) license (http:/ / creativecommons.org/licenses/by/4.0/). 